Maltepe Journal of Mathematics

ISSN:2667-7660, URL:HTTP://DERGIPARK.ORG.TR/TR/PUB/MJM

Volume III Issue 2 (2021), Pages 91-100. Doi: HTtPs://DOI.ORG/10.47087/MJM.930045

\title{
THE CAUCHY PROBLEM OF A PERIODIC KAWAHARA EQUATION IN ANALYTIC GEVREY SPACES
}

\author{
AISSA BOUKAROU*, KADDOUR GUERBATI** \\ *LABORATOIRE DE MATHÉMATIQUES ET SCIENCES APPLIQUÉES UNIVERSITÉ DE \\ GHARDAIA, ALGERIE, ORCID ID: 0000-0002-8586-1058 \\ **LABORATOIRE DE MATHÉMATIQUES ET SCIENCES APPLIQUÉES UNIVERSITÉ DE \\ GHARDAIA, ALGERIE, ORCID ID:: 0000-0003-4256-3760
}

\begin{abstract}
The Cauchy problem for the Kawahara equation with data in analytic Gevrey spaces on the circle is considered and its local well-posedness in these spaces is proved. Using Bourgain-Gevrey type analytic spaces and appropriate bilinear estimates, it is shown that local in time wellposedness holds when the initial data belong to an analytic Gevrey spaces of order $\sigma$. Moreover, the solution is not necessarily $G^{\sigma}$ in time. However, it belongs to $G^{5 \sigma}$ near zero for every $x$ on the circle.
\end{abstract}

\section{Introduction, RELATED RESUlts AND POSITION PROBLEM}

The shallow water equations describes the flow below a pressure surface in a fluid. They are PDEs of hyperbolic type (or parabolic if we consider viscous shear). For $x \in \mathbb{T}, t \in \mathbb{R}$, we denote by $u=u(x, t)$. When we write $(1.1)$, we mean the equation number $i$ from the problem (1.1) subjected with the initial data $u(x, 0)=u_{0}(x)$. We consider

$$
\left\{\begin{array}{l}
\partial_{t} u+\alpha \partial_{x}^{5} u+\beta \partial_{x}^{3} u+\gamma \partial_{x} u+\mu \partial_{x}\left(u^{2}\right)=0, \\
u(x, 0)=u_{0}(x)
\end{array}\right.
$$

here the parameters $\alpha \neq 0, \beta$ and $\gamma$ are real numbers and $\mu$ is a complex number . To outline our contributions, we will extend the results in 2 and 23 , where the solution was obtained in $X_{s, b}$ to the analytic Gevrey-Bourgain spaces $X_{\sigma, \delta, s, b}$ with also regularity in time.

So, from the mathematical point of view, it is important to study the wellposedness and time regularity for the shallow water equations which happens in the water waves with surface tension, in which the Bond number takes on the critical value (See [3], [5], 6], 8]).

Recently, Y. Jia and Z. Huo 2] considered a Cauchy problems (1.1), the authors obtained local well-posedness for data in $H^{s}(\mathbb{R})$ with $s>-7 / 4$ for $\partial_{x} u^{2}$.

2010 Mathematics Subject Classification. Primary: 35E15 ; Secondaries: 35Q53

Key words and phrases. Kawahara equation; Well-posedness; Analytic Gevrey spaces; Bourgain spaces; Bilinear estimates; Time regularity.

(C)2019 Maltepe Journal of Mathematics.

Submitted on April 30 th, 2021. Published on October 30th, 2021

Communicated by Ibrahim CANAK, and Sefa Anl SEZER.. 
Motivated by all the above papers, we investigate the well-posedness of 1.1 in Analytic Gevry spaces to extend results in 23 . The second novelty located in the study of Gevrey's temporal regularity for the unique solution, inspired and motivated by [3] and [5] on the temporal regularity of solutions to KdV-type equations with analytical data of Gevrey.

We begin by presenting some ideas to get the well-posedness, we are working mainly on the integral equivalent formulation of (1.1) as

$$
u=S(t) u_{0}-\int_{0}^{t} S\left(t-t^{\prime}\right) \partial_{x} u^{2}\left(t^{\prime}\right) d t^{\prime},
$$

where the unit operator related to the corresponding linear equation is

$$
S(t)=\mathcal{F}_{x}^{-1} e^{-i t\left(\alpha \xi^{5}-\beta \xi^{3}+\gamma \xi\right)} \mathcal{F}_{x} .
$$

Let us define the phase function as follows

$$
\phi(\xi)=\alpha \xi^{5}-\beta \xi^{3}+\gamma \xi,
$$

We define the needed spaces beginning by the spaces of analytic Gevrey functions that contain our initial data. For $s \in \mathbb{R}, \delta>0$ and $\sigma \geq 1$, let

$$
G^{\sigma, \delta, s}(\mathbb{T})=\left\{f \in L^{2}(\mathbb{T}) ;\|f\|_{G^{\sigma, \delta, s}(\mathbb{T})}^{2}=\sum_{k \in \mathbb{Z}} e^{2 \delta|k|^{1 / \sigma}}\langle k\rangle^{2 s}|\widehat{f}(k)|^{2}<\infty\right\},
$$

where $\langle\cdot\rangle=\left(1+|\cdot|^{2}\right)^{1 / 2}$.

For $\delta=0$, the space $G^{\sigma, \delta, s}(\mathbb{T})$ coincides with the standard Sobolev space $H^{s}(\mathbb{T})$.

We then define the analytic Gevrey -Bourgain spaces related to Kawahara equation. The completion of the Schwartz class $S(\mathbb{T} \times \mathbb{R})$ is given by $X_{\sigma, \delta, s, b}(\mathbb{T} \times$ $\mathbb{R})\left(\operatorname{resp} . Y_{\sigma, \delta, s, b}\right)$, for $s, b \in \mathbb{R}, \delta>0$ and $\sigma \geq 1$, subjected to the norm

$$
\begin{aligned}
\|u\|_{X_{\sigma, \delta, s, b}(\mathbb{T} \times \mathbb{R})} & =\left(\sum_{k \in \mathbb{Z}} \int e^{2 \delta|k|^{1 / \sigma}}\langle k\rangle^{2 s}\langle\tau+\phi(k)\rangle^{2 b}|\widehat{u}(k, \tau)|^{2} d \tau\right)^{\frac{1}{2}} . \\
\|u\|_{Y_{\sigma, \delta, s, b}(\mathbb{T} \times \mathbb{R})} & =\left(\sum_{k \in \mathbb{Z}}\left(\int e^{\delta|k|^{1 / \sigma}}\langle k\rangle^{s}\langle\tau+\phi(k)\rangle^{b}|\widehat{u}(k, \tau)| d \tau\right)^{2}\right)^{\frac{1}{2}} .
\end{aligned}
$$

In addition, let

$$
Z_{\sigma, \delta, s, b}=X_{\sigma, \delta, s, b} \cap Y_{\sigma, \delta, s, b-1 / 2}
$$

be the Banach space endowed with the norm

$$
\|u\|_{Z_{\sigma, \delta, s, b}(\mathbb{T} \times \mathbb{R})}=\|u\|_{X_{\sigma, \delta, s, b}(\mathbb{T} \times \mathbb{R})}+\|u\|_{Y_{\sigma, \delta, s, b-1 / 2}(\mathbb{T} \times \mathbb{R})} .
$$

For $\delta=0$, the space $Z_{\sigma, \delta, s, b}=X_{\sigma, \delta, s, b} \cap Y_{\sigma, \delta, s, b-1 / 2}$ coincides with the standard Bourgain type space $Z_{s, b}=X_{s, b} \cap Y_{s, b-1 / 2}$.

We organize this paper as follows. In Section 2, our main results regarding the well-posedness (Theorem 2.1) and regularity (Theorem 2.2) in the analytic GevreyBourgain spaces for 1.1) are stated. In Section 3, all Theorems by deriving the bilinear estimates are proved in details. 


\section{MAIN RESUlts}

Theorem 2.1. Let $s>0, \sigma \geqslant 1, \delta>0$ and $u_{0} \in G^{\sigma, \delta, s}(\mathbb{T})$. Then for some real number $b>\frac{1}{2}$, which is near enough to $\frac{1}{2}$, and a constant $T>0$, such that (1.1) admits a unique local solution $u \in C\left([0, T], G^{\sigma, \delta, s}(\mathbb{T})\right) \cap Z_{\sigma, \delta, s, \frac{1}{2}}$. Moreover, given $t \in(0, T)$, the map $u_{0} \rightarrow u(t)$ is Lipschitz continuous from $G^{\sigma, \delta, s}(\mathbb{T})$ to $C\left([0, T], G^{\sigma, \delta, s}(\mathbb{T})\right)$.

Our next goal is to study Gevrey's temporal regularity of the unique solution obtained in Theorem 2.1. A periodic function $f(x)$ is the Gevrey class of order $\sigma$, if there exists a constant $C>0$ such that

$$
\sup _{x \in \mathbb{T}}\left|\partial_{x}^{l} f(x)\right| \leq C^{l+1}(l !)^{\sigma} \quad l=0,1,2, \ldots
$$

Theorem 2.2. Let $s>0, \sigma \geqslant 1, \delta>0$ and $\beta=\gamma=\mu=\alpha=1$. If $u_{0} \in G^{\sigma, \delta, s}(\mathbb{T})$, then the solution $u \in C\left([0, T], G^{\sigma, \delta, s}(\mathbb{T})\right)$ given by Theorem 2.1 belongs to the Gevrey class $G^{5 \sigma}$ in time variable.

\section{Proof of MAIN TheOrems}

We are going to prepare the prove of our main theorems, let us beginning by the embedded result in the next lemma, which is useful for Theorem 2.1.

Lemma 3.1. Let $s \in \mathbb{R}, \sigma \geqslant 1$ and $\delta>0$, we have

$$
Z_{\sigma, \delta, s, \frac{1}{2}}(\mathbb{T} \times \mathbb{R}) \hookrightarrow C\left([0, T], G^{\sigma, \delta, s}(\mathbb{T})\right)
$$

3.1. Existence of solution. Taking the Fourier transform with respect to $x$ of the Cauchy problems (1.1), after an ordinary calculation, we get

$$
u=S(t) u_{0}-\int_{0}^{t} S\left(t-t^{\prime}\right) \partial_{x} u^{2}\left(t^{\prime}\right) d t^{\prime},
$$

we localize it $t$ by using a cut-off function, satisfying $\psi \in C_{0}^{\infty}(\mathbb{R})$, with $\psi=1$ in $\left[-\frac{1}{2}, \frac{1}{2}\right]$ and $\operatorname{supp} \psi \subset[-1,1]$. We consider the operator $\Phi u$ given by

$$
\Phi(u)=\psi(t) S(t) u_{0}-\psi(t) \int_{0}^{t} S\left(t-t^{\prime}\right) \partial_{x} u^{2}\left(t^{\prime}\right) d t^{\prime},
$$

We now estimate the fist part in the right hand side of (3.1).

Lemma 3.2. Let $s \in \mathbb{R}, \delta>0$ and $\sigma \geqslant 1$, for some constant $C>0$, we have

$$
\left\|\psi(t) S(t) u_{0}\right\|_{Z_{\sigma, \delta, s, b}(\mathbb{T} \times \mathbb{R})} \leq C\left\|u_{0}\right\|_{G^{\sigma, \delta, s}(\mathbb{T})},
$$

for all $u_{0} \in G^{\sigma, \delta, s}(\mathbb{T})$.

Proof. Define the operator $A$ defined by

$$
\widehat{A u}^{x}(k, t)=e^{\delta|k|^{1 / \sigma}} \widehat{u}^{x}(k, t)
$$

for $\delta=0$ can be found in Lemma 2.1 of [23]. These inequalities clearly remain valid for $\delta>0$, as one merely has to replace $u_{0}$ by $A u_{0}$ in these results.

We estimate the second part in right hand side of (3.1). 
Lemma 3.3. Let $s \in \mathbb{R}, \delta>0$ and $\sigma \geqslant 1$, then for some constant $C>0$, we have

$$
\left\|\psi(t) \int_{0}^{t} S\left(t-t^{\prime}\right) F\left(x, t^{\prime}\right) \mathrm{d} t^{\prime}\right\|_{Z_{\sigma, \delta, s, \frac{1}{2}}}(\mathbb{T} \times \mathbb{R}) \leq C\|F\|_{Z_{\sigma, \delta, s,-\frac{1}{2}}}(\mathbb{T} \times \mathbb{R}) .
$$

Proof. Define $U=\psi_{T}(t) \int_{0}^{t} S\left(t-t^{\prime}\right) F\left(x, t^{\prime}\right) \mathrm{d} t^{\prime}$ and using the operator $A$.

$$
\begin{aligned}
\widehat{A U}^{x}(k, t) & =\psi(t) \int_{0}^{t}\left(e^{-i\left(t-t^{\prime}\right) \phi(k)}\right) e^{\delta|k|^{1 / \sigma}} \widehat{F}^{x}\left(k, t^{\prime}\right) \mathrm{d} t^{\prime} \\
& =\psi(t) \int_{0}^{t}\left[S\left(\widehat{\left.-t^{\prime}\right)(A F)}\right]^{x}\left(k, t^{\prime}\right) \mathrm{d} t^{\prime}\right.
\end{aligned}
$$

Thus,

$$
\|U\|_{Z_{\sigma, \delta, s, \frac{1}{2}}(\mathbb{T} \times \mathbb{R})}=\|A U\|_{Z_{s, \frac{1}{2}}(\mathbb{T} \times \mathbb{R})}=\left\|\psi(t) \int_{0}^{t} S\left(t-t^{\prime}\right) A F\left(x, t^{\prime}\right) d t^{\prime}\right\|_{Z_{s, \frac{1}{2}}(\mathbb{T} \times \mathbb{R}) .} .
$$

Using Lemma 2.1. in 23], we have

$\left\|\psi(t) \int_{0}^{t} S\left(t-t^{\prime}\right) A F\left(x, t^{\prime}\right) d t^{\prime}\right\|_{Z_{s, \frac{1}{2}}(\mathbb{T} \times \mathbb{R})} \leq C\|A F\|_{Z_{s,-\frac{1}{2}}(\mathbb{T} \times \mathbb{R})}=C\|F\|_{Z_{\sigma, \delta, s,-\frac{1}{2}}(\mathbb{T} \times \mathbb{R})}$.

In order to treat the different nonlinear terms, we will see several lemmas. Here the bilinear estimate is given in the next lemma.

Lemma 3.4. If $s>0$, let $\sigma \geqslant 1, \delta>0$. Then

$$
\left\|\partial_{x}\left(u_{1} u_{2}\right)\right\|_{Z_{\sigma, \delta, s,-\frac{1}{2}}(\mathbb{T} \times \mathbb{R})} \leqslant C\left\|u_{1}\right\|_{Z_{\sigma, \delta, s, \frac{1}{2}}(\mathbb{T} \times \mathbb{R})}\left\|u_{2}\right\|_{Z_{\sigma, \delta, s, \frac{1}{2}}(\mathbb{T} \times \mathbb{R}) .} .
$$

Proof. We observe, by considering the operator $A$ in (3.3), that

$$
\begin{aligned}
e^{\delta|k|^{1 / \sigma} \widehat{u_{1} u_{2}}} & =(2 \pi)^{-2} e^{\delta|k|^{1 / \sigma} \widehat{u_{1}} * \widehat{u_{2}}} \\
& \leqslant(2 \pi)^{-2} \int_{\mathbb{R}^{2}} e^{\delta|k-\eta|^{1 / \sigma}} \widehat{u_{1}}(k-\eta, \tau-\rho) e^{\delta|\eta|^{1 / \sigma} \widehat{u_{2}}(\eta, \rho) d \eta d \rho} \\
& =\widehat{A u_{1} A u_{2}},
\end{aligned}
$$

since $\delta|k|^{1 / \sigma} \leqslant \delta|k-\eta|^{1 / \sigma}+\delta|\eta|^{1 / \sigma}, \quad \forall \sigma \geqslant 1$. Then

$$
\begin{aligned}
\left\|\partial_{x}\left(u_{1} u_{2}\right)\right\|_{Z_{\sigma, \delta, s,-\frac{1}{2}}(\mathbb{T} \times \mathbb{R})} & =\left\|\partial_{x}\left(A\left(u_{1} u_{2}\right)\right)\right\|_{Z_{s,-\frac{1}{2}}(\mathbb{T} \times \mathbb{R})} \\
& \leq\left\|\partial_{x}\left(A u_{1} A u_{2}\right)\right\|_{Z_{s,-\frac{1}{2}}}(\mathbb{T} \times \mathbb{R}) .
\end{aligned}
$$

Now, by using Lemma 2.3. of [23], there exists $C>0$ such that

$$
\begin{aligned}
\left\|\partial_{x}\left(A u_{1} A u_{2}\right)\right\|_{Z_{s,-\frac{1}{2}}(\mathbb{T} \times \mathbb{R})} & \leqslant C\left\|A u_{1}\right\|_{Z_{s, \frac{1}{2}}(\mathbb{T} \times \mathbb{R})}\left\|A u_{2}\right\|_{Z_{s, \frac{1}{2}}(\mathbb{T} \times \mathbb{R})} \\
& =C\left\|u_{1}\right\|_{Z_{\sigma, \delta, s, \frac{1}{2}}(\mathbb{T} \times \mathbb{R})}\left\|u_{2}\right\|_{Z_{\sigma, \delta, s, \frac{1}{2}}(\mathbb{T} \times \mathbb{R}) .} .
\end{aligned}
$$

We are now ready to estimate all the terms in (3.1) by using the bilinear estimates in the above lemmas. 
Lemma 3.5. Let $s>0$, and $\sigma \geq 1, \delta>0$. Then, for all $u_{0} \in G^{\sigma, \delta, s}(\mathbb{T})$, with some constant $C>0$, we have

$$
\|\Phi(u)\|_{Z_{\sigma, \delta, s, \frac{1}{2}}(\mathbb{T} \times \mathbb{R})} \leq C\left(\left\|u_{0}\right\|_{G^{\sigma, \delta, s}(\mathbb{T})}+\|u\|_{Z_{\sigma, \delta, s, \frac{1}{2}}^{2}(\mathbb{T} \times \mathbb{R})}\right),
$$

and

$$
\|\Phi(u)-\Phi(v)\|_{Z_{\sigma, \delta, s, \frac{1}{2}}(\mathbb{T} \times \mathbb{R})} \leq C\|u-v\|_{Z_{\sigma, \delta, s, \frac{1}{2}}(\mathbb{T} \times \mathbb{R})}\|u+v\|_{Z_{\sigma, \delta, s, \frac{1}{2}}(\mathbb{T} \times \mathbb{R})},
$$

for all $u, v \in Z_{\sigma, \delta, s, \frac{1}{2}}(\mathbb{T} \times \mathbb{R})$

Proof. To prove estimate (3.7), we follow

$$
\begin{aligned}
&\|\Phi(u)\|_{Z_{\sigma, \delta, s, \frac{1}{2}}(\mathbb{T} \times \mathbb{R})} \leq\left\|\psi_{T}(t) S(t) u_{0}\right\|_{Z_{\sigma, \delta, s, \frac{1}{2}}(\mathbb{T} \times \mathbb{R})} \\
&+\left\|\psi_{T}(t) \int_{0}^{t} S\left(t-t^{\prime}\right) \partial_{x} u^{2}\left(t^{\prime}\right) d t^{\prime}\right\|_{Z_{\sigma, \delta, s, \frac{1}{2}}(\mathbb{T} \times \mathbb{R})} \\
& \leq C\left\|u_{0}\right\|_{G^{\sigma, \delta, s}(\mathbb{T})}+C\left\|\partial_{x} u^{2}\right\|_{Z_{\sigma, \delta, s,-\frac{1}{2}}(\mathbb{T} \times \mathbb{R})} \\
& \leq C\left\|u_{0}\right\|_{G^{\sigma, \delta, s}(\mathbb{T})}+C\|u\|_{Z_{\sigma, \delta, s, \frac{1}{2}}(\mathbb{T} \times \mathbb{R})} .
\end{aligned}
$$

For the estimate 3.8$)$, we observe that

$$
\Phi(u)-\Phi(v)=\psi_{T}(t) \int_{0}^{t} S\left(t-t^{\prime}\right)\left(\partial_{x} u^{2}-\partial_{x} v^{2}\right)\left(x, t^{\prime}\right) d t^{\prime},
$$

where $\omega=\partial_{x} u^{2}-\partial_{x} v^{2}$ is now given by

$$
\omega=\partial_{x}\left(u^{2}-v^{2}\right)=\partial_{x}[(u+v)(u-v)],
$$

Thus, from the previous results, we obtain (3.8).

We will show that the map $\Phi$ is a contraction on the ball $\mathbb{B}(0, r)$ to $\mathbb{B}(0, r)$. where $u_{0}$ satisfies the smallness condition $\left\|u_{0}\right\|_{G^{\sigma, \delta, s}(\mathbb{T})} \leq \frac{1}{18 C^{2}}$ and $r=\frac{1}{6 C}$

Lemma 3.6. Let $s>0$ and $\sigma \geq 1$. Then, for all $u_{0} \in G^{\sigma, \delta, s}(\mathbb{T})$, such that the map $\Phi: \mathbb{B}(0, r) \rightarrow \mathbb{B}(0, r)$ is a contraction, where $\mathbb{B}(0, r)$ is given by

$$
\mathbb{B}(0, r)=\left\{u \in Z_{\sigma, \delta, s, \frac{1}{2}}(\mathbb{T} \times \mathbb{R}) ;\|u\|_{Z_{\sigma, \delta, s, \frac{1}{2}}(\mathbb{T} \times \mathbb{R})} \leq r\right\} .
$$

Proof. To prove Lemma 3.6 we need to use Lemma 3.5 .

This completes the prove of existence Theorem 2.1.

3.2. Continuous dependence of the initial data. To prove continuous dependence of the initial data in $Z_{\sigma, \delta, s, \frac{1}{2}}(\mathbb{T} \times \mathbb{R})$ we will prove the following.

Lemma 3.7. Let $s>0$ and $\sigma \geq 1, \delta>0$. Then for all $u_{0}, v_{0} \in G^{\sigma, \delta, s}(\mathbb{T})$, if $u$ and $v$ are two solutions to (1.1) corresponding to initial data $u_{0}$ and $v_{0}$. We have

$$
\|u-v\|_{C\left([0, T], G^{\sigma, \delta, s}(\mathbb{T})\right)} \leq 2 C_{0} C\left\|u_{0}-v_{0}\right\|_{G^{\sigma, \delta, s}(\mathbb{T})} .
$$

Proof. To prove Lemma 3.7 we need to use Lemma3.1.

This completes the prove of Theorem 2.1 


\subsection{Time regularity.}

Lemma 3.8. (Proposition 3.1, 7] ) Let $s>0$, and let $\delta>0, \sigma \geq 1, u \in$ $C\left([0, T] ; G^{\sigma, \delta, s}(\mathbb{T})\right)$ be the solution of $[1.1)$. Then $u \in G^{\sigma}$ in $x, \forall t \in[0, T]$, i.e., for some $C>0$, we have

$$
\left|\partial_{x}^{l} u\right| \leqslant C^{l+1}(l !)^{\sigma}, l \in\{0,1, \ldots\}, \quad \forall x \in \mathbb{T}, t \in[0, T] .
$$

In this section, we shall prove the time regularity of the solution as stated in Theorem 2.1 on the circle. The proof on the line is analogous.

Let us consider as in [3], for $\epsilon>0$, the sequences

$$
m_{q}=\frac{c(q !)^{\sigma}}{(q+1)^{2}},(q=0,1,2, \ldots)
$$

and

$$
M_{q}=\epsilon^{1-q} m_{q}, \epsilon>0 \text { and }(q=1,2,3, \ldots) \text {, }
$$

where $c$ will be chosen (see [1]) so that the next inequality holds

$$
\sum_{0 \leq l \leq k}\left(\begin{array}{l}
k \\
l
\end{array}\right) m_{l} m_{k-l} \leqslant m_{k}
$$

Removing the endpoints 0 and $k$ in the left hand side of $(3.13)$ and using the sequence $M_{q}$, we obtain

$$
\sum_{0<l<k}\left(\begin{array}{l}
k \\
l
\end{array}\right) M_{l} M_{k-l} \leq M_{k}, \forall \epsilon>0 .
$$

Next, one can check that for any $\epsilon>0$ the sequence $M_{q}$ satisfies the following inequality

$$
M_{j} \leqslant \epsilon M_{j+1}, \text { for } j \geq 2 \text {. }
$$

Also, one can check that for a given $C>1$, there exists $\epsilon_{0}>0$ such that for any $0<\epsilon \leqslant \epsilon_{0}$ we have

$$
C^{j+1} j ! \leqslant M_{j}, \text { for } j \geq 2 .
$$

By the definition of $M_{1}$ and $M_{2}$ in (3.12), we have for $j=1$, that

$$
M_{1}=a \epsilon M_{2}, \text { where } a=\frac{9}{4(2 !)^{\sigma}},
$$

for some $C>0$. Also, we define the following constants

$$
M_{0}=\frac{c}{8} \text { and } M=\max \left\{2, \frac{8 C}{c}, \frac{4 C^{2}}{c}\right\} .
$$

The next lemma is the main idea for the proof of Theorem 2.2

Lemma 3.9. Let $u$ be the solution of (1.1) satisfying (3.10), then there exists $\epsilon_{0}>0$ such that for any $0<\epsilon \leqslant \epsilon_{0}$ we have

$$
\left|\partial_{t}^{j} \partial_{x}^{l} u\right| \leqslant M^{2 j+1} M_{l+5 j}, j \in\{0,1,2, \ldots\}, l \in\{0,1,2, \ldots\},
$$

for all $x \in \mathbb{T}, t \in[0, T]$.

Proof. (Of Lemma 3.9)

We will prove 3.18 by induction. Let $j=0$, for $l=0$, it follows from 3.10 and the definition of $M$ in 3.17 that

$$
|u| \leq C \leq M M_{0}, \quad \forall x \in \mathbb{T}, t \in[0, T] .
$$


Similarly, for $l=1$, we have

$$
\left|\partial_{x} u\right| \leq C^{2} \leqslant M M_{1}, \forall x \in \mathbb{T}, t \in[0, T] .
$$

By (3.10) and (3.16), for $l \geq 2$, there exists $\epsilon_{0}>0$ such that for any $0<\epsilon \leqslant \epsilon_{0}$, we have

$$
\left|\partial_{x}^{l} u\right| \leq C^{l+1}(l !)^{\sigma} \leq M_{l} \leq M M_{l}, \forall x \in \mathbb{T}, t \in[0, T] .
$$

This completes the proof of $(3.18)$ for $j=0$ and $l \in\{0,1, \ldots\}$.

Next, we will assume that $(3.18)$ is true for $0 \leq q \leq j$ and $l \in\{0,1, \ldots\}$ and we will prove it for $q=j+1$ and $l \in\{0,1, \ldots\}$.

We begin by noting that

$$
\left|\partial_{t}^{j+1} \partial_{x}^{l} u\right|=\left|\partial_{t}^{j} \partial_{x}^{l}\left(\partial_{t} u\right)\right| \leqslant\left|\partial_{t}^{j} \partial_{x}^{l+5} u\right|+\left|\partial_{t}^{j} \partial_{x}^{l+3} u\right|+\left|\partial_{t}^{j} \partial_{x}^{l+1} u\right|+\left|\partial_{t}^{j} \partial_{x}^{l}\left(\partial_{x} u^{2}\right)\right| .
$$

Using the induction hypotheses and the condition $M>2$, we estimate the second term $\partial_{t}^{j} \partial_{x}^{l+5} u, \partial_{t}^{j} \partial_{x}^{l+3} u$ and $\partial_{t}^{j} \partial_{x}^{l+1} u$ as follows

$$
\begin{aligned}
\left|\partial_{t}^{j} \partial_{x}^{l+5} u\right| & \leqslant M^{2 j+1} M_{l+5+5 j}=M^{-2} M^{2(j+1)+1} M_{l+5(j+1)}, \\
& \leqslant \frac{1}{4} M^{2(j+1)+1} M_{l+5(j+1)},
\end{aligned}
$$

and

$$
\begin{aligned}
\left|\partial_{t}^{j} \partial_{x}^{l+3} u\right| & \leqslant M^{2 j+1} M_{l+3+5 j}=M^{-2} M^{2(j+1)+1} M_{l+5 j+3)}, \\
& \leqslant \frac{\epsilon^{2}}{4} M^{2(j+1)+1} M_{l+5(j+1)},
\end{aligned}
$$

and

$$
\left|\partial_{t}^{j} \partial_{x}^{l+1} u\right| \leqslant M^{2 j+1} M_{l+1+5 j} \leqslant \frac{\epsilon^{4}}{4} M^{2(j+1)+1} M_{l+5(j+1)} .
$$

All this estimates are taken for the linear terms. For the nonlinear term $\left(\partial_{x} u^{2}\right)$, using Leibniz's rule twice and the induction hypothesis, we have a different cases. We need the next results.

Lemma 3.10. (3]) Given $n, k \in\{0,1,2, \ldots\}$ we have

$$
\sum_{p=0}^{n} \sum_{q=0}^{k}\left(\begin{array}{l}
n \\
p
\end{array}\right)\left(\begin{array}{l}
k \\
q
\end{array}\right) L_{(n-p)+5(k-q)} L_{p+5 q} \leqslant \sum_{r=1}^{m}\left(\begin{array}{c}
m \\
r
\end{array}\right) L_{r} L_{m-r},
$$

where $L_{j}, j=0,1, \ldots, m$ positive real numbers with $m=n+5 k$

$$
\begin{aligned}
\left|\partial_{t}^{j} \partial_{x}^{l+1}\left(u^{2}\right)\right| & \leqslant \sum_{p=0}^{l+1} \sum_{q=0}^{j}\left(\begin{array}{c}
l+1 \\
p
\end{array}\right)\left(\begin{array}{l}
j \\
p
\end{array}\right)\left|\partial_{t}^{j-q} \partial_{x}^{l+1-p} u \| \partial_{t}^{q} \partial_{x}^{p} u\right| \\
& \leqslant \sum_{p=0}^{l+1} \sum_{q=0}^{j}\left(\begin{array}{c}
l+1 \\
p
\end{array}\right)\left(\begin{array}{c}
j \\
p
\end{array}\right) M^{2(j-q)+1} M_{l+1-p+5(j-q)} M^{2 q+1} M_{p+5 q} \\
& =M^{2(j+1)} \sum_{p=0}^{l+1} \sum_{q=0}^{j}\left(\begin{array}{c}
l+1 \\
p
\end{array}\right)\left(\begin{array}{l}
j \\
p
\end{array}\right) M_{l+1-p+5(j-q)} M_{p+5 q} .
\end{aligned}
$$


Next, using Lemma 3.10 , with $n=l+1, k=j, L_{j}=M_{j}, m=l+1+5 j$, we obtain

$$
\begin{aligned}
& \sum_{p=0}^{l+1} \sum_{q=0}^{j}\left(\begin{array}{c}
l+1 \\
p
\end{array}\right)\left(\begin{array}{l}
j \\
p
\end{array}\right) M_{l+1-p+5(j-q)} M_{p+5 q}, \\
& \leqslant \sum_{r=1}^{m}\left(\begin{array}{c}
m \\
r
\end{array}\right) L_{r} L_{m-r} \leq\left(M_{0}+\epsilon\right) M_{m}, \\
& =\left(M_{0}+\epsilon\right) M_{l+5 j+1},
\end{aligned}
$$

then

$$
\begin{aligned}
\left|\partial_{t}^{j} \partial_{x}^{l+1}\left(u^{2}\right)\right| & \leqslant M^{2(j+1)}\left(M_{0}+\epsilon\right) M_{l+5 j+1}, \\
& \leqslant M^{-2} M^{2(j+1)+1} \epsilon^{4}\left(M_{0}+\epsilon\right) M_{l+5(j+1)}, \\
& \leqslant \frac{\epsilon^{4}\left(M_{0}+\epsilon\right)}{4} M^{2(j+1)+1} M_{l+5(j+1)} .
\end{aligned}
$$

Noting that in the last inequality we have used the fact that $l+5 j+1 \geq 2$, since we are assuming that either $j \neq 0$ or $l \neq 0$.

Now, choosing $\epsilon \leq \epsilon_{0}=\left(\frac{1}{\left(M_{0}+\epsilon\right)}\right)^{\frac{1}{4}}<1$ to obtain

$$
\epsilon^{4}\left(M_{0}+\epsilon\right) \leq \epsilon^{4}\left(M_{0}+1\right) \leq\left(M_{0}+1\right)\left(\frac{1}{\left(M_{0}+1\right)}\right)=1
$$

Hence,

$$
\left|\partial_{t}^{j} \partial_{x}^{l+1}\left(u^{2}\right)\right| \leq \frac{1}{4} M^{2(j+1)+1} M_{l+5(j+1)}
$$

Which completes the proof.

Proof. (Of Theorem 2.2) By Lemma 3.9, we have

$$
\left|\partial_{t}^{j} \partial_{x}^{l} u\right| \leqslant M^{2 j+1} M_{l+5 j}, j \in\{0,1,2, \ldots\}, l \in\{0,1,2, \ldots\},
$$

where

$$
M_{q}=\epsilon^{1-q} \frac{c(q !)^{\sigma}}{(q+1)^{2}}, q=1,2, \ldots
$$

Applying this inequality for $j \in\{1,2, \ldots\}$ and $l=0$ gives

$$
\begin{aligned}
\left|\partial_{t}^{j} u\right| & \leqslant M^{2 j+1} M_{5 j}=M M^{2 j} \epsilon^{1-5 j} \frac{c((5 j) !)^{\sigma}}{(5 j+1)^{2}} \\
& \leqslant M \epsilon c\left(\frac{M^{2}}{\epsilon^{5}}\right)^{j}((5 j) !)^{\sigma} \\
& \leqslant L_{0} L^{j}((5 j) !)^{\sigma} \\
& \leqslant L_{0} L^{j} A^{5 \sigma j}\left((j !)^{5}\right)^{\sigma} \\
& \leqslant A_{0}^{j+1}(j !)^{5 \sigma}
\end{aligned}
$$


where $L_{0}=M \epsilon c, L=\frac{M^{2}}{\epsilon^{5}}$ since $(5 j) ! \leqslant A^{5 j}(j !)^{5}$ for $A>0$ and $A_{0}=\max \left\{L_{0}, L A^{5 \sigma}\right\}$. We also have from (3.18) for $l=j=0$, that

$$
|u| \leq M M_{0}=M \frac{c}{8}, \quad \forall x \in \mathbb{T}, t \in[0, T] .
$$

Setting $C=\max \left\{M \frac{c}{8}, A_{0}\right\}$, it follows from 3.24 and 3.25 that for $j \in\{0,1,2, \ldots\}$, we have

$$
\left|\partial_{t}^{j} u\right| \leqslant C^{j+1}(j !)^{5 \sigma}, \quad \forall x \in \mathbb{T}, t \in[0, T] .
$$

Hence, $u \in G^{5 \sigma}$ in $t$.

Which completes the proof of Theorem 2.2 .

Acknowledgments. The authors wish to thank deeply the anonymous referee for his/here useful remarks and his/here careful reading of the proofs presented in this paper.

\section{REFERENCES}

[1] S. Alinhac and G. Metivier, Propagation de l'analyticite des solutions des systmes hyperboliques non-linéaires, Invent. Math., 75, 189-204, 1984.

[2] Y. Jia and Z. Huo, Well-posedness for the fifth-order shallow water equations, J. Differ. Equ., 246, 2448-2467, 2009.

[3] H. Hannah, A. Himonas, G. Petronilho, Gevrey regularity of the periodic gKdV equation, J. Differ. Equ., 250, 2581-2600, 2011.

[4] Z. Huoa and B. Guo, Well-posedness of the Cauchy problem for the Hirota equation in Sobolev spaces $H^{s}$, Nonlinear Analysis, 60, 1093-1110, 2005.

[5] J. Gorsky, A. Himonas, C. Holliman, G. Petronilho, The Cauchy problem of a periodic higher order KdV equation in analytic Gevrey spaces, J. Math. Anal. Appl., 405, 349-361, 2013.

[6] C.E. Kenig, G. Ponce, L. Vega, The Cauchy problem for the Kortewegde Vries equation in Sobolev spaces of negative indices, Duke Math. J., 71, 1-21, 1993.

[7] Barostichi R. F., Figueira R. O., Himonas A. A., Well-posedness of the "good" Boussinesq equation in analytic Gevrey spaces and time regularity, J. Diff. Equ., 267(5), 2019, 31813198.

[8] C.E. Kenig, G. Ponce, L. Vega, A bilinear estimate with applications to the KdV equation, J. Amer. Math. Soc., 9, 573-603, 1996.

[9] X. Yang, Y. Li, Global well-posedness for a fifth-order shallow water equation in Sobolev spaces, J. Diff. Equ., 248, 1458-1472, 2010.

[10] Z. Zhang, Z. Liu. M, Sun and S. Li, Low Regularity for the Higher Order Nonlinear Dispersive Equation in Sobolev Spaces of Negative Index, J. Dyn. Diff. Equ., 31, 419-433, 2019.

[11] A. Boukarou, Kh. Zennir, K. Guerbati and S. G. Georgiev, Well-posedness of the Cauchy problem of Ostrovsky equation in analytic Gevrey spaces and time regularity, Rend. Circ. Mat. Palermo (2), (2020) https://doi.org/10.1007/s12215-020-00504-7.

[12] A. Boukarou, K. Guerbati, Kh. Zennir, S. Alodhaibi and S. Alkhalaf, Well-Posedness and Time Regularity for a System of Modified Korteweg-de Vries-Type Equations in Analytic Gevrey Spaces, Mathematics 2020, 8, 809.

[13] A. Boukarou, Kh. Zennir, K. Guerbati and S. G. Georgiev, Well-posedness and regularity of the fifth order Kadomtsev-Petviashvili I equation in the analytic Bourgain spaces, Ann. Univ. Ferrara Sez. VII Sci. Mat. (2020) https://doi.org/10.1007/s11565-020-00340-8.

[14] Aissa Boukarou, Kaddour Guerbati \& Khaled Zennir (2021): Analytic-Gevrey wellposedness of generalized Benjamin-Ono equation, Journal of Interdisciplinary Mathematics, DOI: $10.1080 / 09720502.2021 .1917062$

[15] Aissa Boukarou, Kaddour Guerbati, Khaled Zennir and Mohammad Alnegga (2021): Gevrey regularity for the generalized Kadomtsev-Petviashvili I (gKP-I) equation, AIMS Mathematics 6(9), DOI: $10.3934 /$ math.2021583

[16] B.A. Kupershmidt, A super Kortewegde Vries equation: An integrable system, Phys. Lett. A 102 (56) (1984) 213215. 
[17] A. Boukarou, K. Guerbati, Kh. Zennir, On the radius of spatial analyticity for the higher order nonlinear dispersive equation, Mathematica Bohemica,March 16, 2021. 10.21136/MB.2021.0096-20.

[18] K Zennir, A Boukarou, RN Alkhudhayr Global Well-Posedness for Coupled System of mKdV Equations in Analytic Spaces Journal of Function Spaces, 2021, https://doi.org/10.1155/2021/6614375.

[19] A. Boukarou, K. Guerbati, Kh. Zennir, Local well-posedness and time regularity for a fifthorder shallow water equations in analytic GevreyBourgain spaces. , Monatsh Math 193, 763782 (2020). https://doi.org/10.1007/s00605-020-01464-x

[20] A. Boukarou, D. Oliveira da Silva, Kh. Guerbati and Kh. Zennir, Global well-posedness for the fifth-order Kadomtsev-Petviashvili II equation in anisotropic Gevrey Spaces, http://arXiv:2006.12859.

[21] E.S. Benilov, R. Grimshaw, E.P. Kuznetsova, The generation of radiating waves in a singularly-perturbed Kortewegde Vries equation, Phys. D 69 (34) (1993) 270278.

[22] J.K. Hunter, J. Scheurle, Existence of perturbed solitary wave solutions to a model equation for water waves, Phys. D 32 (2) (1988) 253268.

[23] X Zhao, BY Zhang,Global controllability and stabilizability of Kawahara equation on a periodic domain, Mathematical Control \& Related Fields, 2015, pp. 335-358

Aissa Boukarou,

Laboratoire de Mathématiques et Sciences appliquées Université de Ghardaia, Algerie, ORCID ID: 0000-0002-8586-1058

Email address: boukarouaissa@gmail.com

KadDOur Guerbati,

Laboratoire de Mathématiques et Sciences appliquées Université de Ghardaia, Algerie, ORCID ID:: 0000-0003-4256-3760

Email address: guerbati_k@yahoo.com 